UTTG-13-94

August 18, 2018

\title{
Non-Perturbative Decoupling of Heavy Fermions
}

\author{
Moshe Rozalif \\ Theory Group, Department of Physics \\ University of Texas, Austin, Texas 78712
}

\begin{abstract}
We show that heavy fermions decouple from the low energy physics also in non-perturbative instanton effects. Provided the heavy fermions are lighter than the symmetry breaking scale, all the instanton effects should be expressed as local operators in the effective Lagrangian. The effective theory itself doesn't admit instantons. We present the mechanism which suppresses instantons in the effective theory.
\end{abstract}

\footnotetext{
${ }^{\dagger}$ Research supported in part by the Robert A. Welch Foundation and NSF Grant PHY 9009850
} 


\section{Introduction}

In a recent paper [1] non-perturbative instanton effects were claimed to violate decoupling. Several solutions to the problem were suggested [2, 3]. In this paper we reformulate the problem in the context of the standard model and clarify the the process of integrating out heavy fermions.

In what follows we consider the standard model with three families and inter-family mixing. The color and hypercharge indices are suppressed, and the lepton doublets are left undisplayed, since they are irrelevant for the discussion.

The Lagrangian of the model is (see the appendix for notations):

$\mathcal{L}=-\frac{1}{2} \operatorname{Tr}\left(W_{\mu \nu} W^{\mu \nu}\right)+\frac{1}{2}\left(\partial_{\mu} \tilde{\sigma}\right)^{2}-\frac{\lambda}{4}\left(\tilde{\sigma}^{2}-v^{2}\right)^{2}+\frac{\tilde{\sigma}}{4} \operatorname{Tr}\left(D_{\mu} U^{\dagger} D^{\mu} U\right)+L_{\text {fermionic }}$

For one quark doublet the fermion action is:

$$
\mathcal{L}_{\psi}=i \psi^{\dagger} \sigma^{\mu} D_{\mu} \psi+i \bar{\psi}^{\dagger} \sigma^{\mu} \partial_{\mu} \bar{\psi}-\left(\bar{\psi}^{T} \frac{\mathcal{M}_{q}}{v} \tilde{\sigma} U \psi+\text { c.c. }\right)
$$

We assume equality of the top and bottom mass, $\mathcal{M}_{q}=m_{t} \mathbb{1}$, unless otherwise indicated. $\langle\tilde{\sigma}\rangle=\sigma$ breaks the symmetry, $\sigma \equiv \tilde{\sigma}-v$ is the physical Higgs. $U$ is the angular Higgs (the would-be Goldstone bosons).

In theories with Higgs fields no exact instanton solutions exist. However, there exist approximate solutions, known as "constrained instantons" [4. In the next section we review the constrained instanton construction and the resulting 'tHooft operator [5]. This operator violates baryon number by 3 units.

We integrate out one heavy quark doublet, assuming $m_{\sigma}$ and $m_{W}$ are lighter than $m_{t}$, so the effective theory contains the same bosonic fields. After integrating out the fermions the theory's predictions for low energy processes should be obtainable from a local effective Lagrangian — this is what is meant by "decoupling" in this context. 
First, the effective Lagrangian should reproduce the original 't Hooft operator. This matching is shown in section 3. Secondly, it must predict vanishing amplitudes for $\Delta B=2$ processes, which would naively appear as 't Hooft operators in a two family model. This is shown in detail in Section 4. We present our conclusions in Section 5.

\section{The Constrained Instanton:}

In this section we review instanton effects in the standard model. We follow some of the notation of [6], and the reader is referred to the latter for more details.

Fermion number violating amplitudes arise in a semi-classical expansion around an Euclidean configuration with a non-vanishing winding number. Schematically one has the following Euclidean Lagrangian:

$$
\mathcal{L}_{E}=\psi_{A} M(A) \psi_{B}+B(A)
$$

$\psi_{A}, \psi_{B}$ are Euclidean fermions [6], and $A$ some bosonic fields. Expanding to leading $\hbar$ order around some configuration $A_{0}$ :

$$
\mathcal{L}_{E}=\psi_{A} M\left(A_{0}\right) \psi_{B}+B\left(A_{0}\right)+\text { corrections } .
$$

The minimal non-vanishing amplitude is then:

$$
\left\langle\psi_{1}\left(x_{1}\right) \ldots \psi_{r}\left(x_{r}\right)\right\rangle=C e^{-S_{0}} \psi_{0}^{1}\left(x_{1}\right) \ldots \psi_{0}^{r}\left(x_{r}\right)
$$

$C$ is a constant calculated from the bosonic fluctuations around $A_{0}, S_{0}=$ $\int d^{4} x B\left(A_{0}\right)$ has to be finite to contribute to the path integral. $r$ is the number of zero modes of the operator $M\left(A_{0}\right)$, related to chiral anomalies by index theorems.

In the case considered here, the relevant configuration is the constrained instanton [4], which is an approximate saddle point with a finite action. After 
an Euclidean rotation and rescaling, the bosonic action is:

$$
\mathcal{L}_{B}=\frac{1}{g^{2}}\left[\frac{1}{2} \operatorname{Tr}\left(W_{\mu \nu} W_{\mu \nu}\right)+\kappa^{2} \operatorname{Tr}\left(D_{\nu} M^{\dagger} D_{\nu} M\right)+\kappa^{2}\left(\tilde{\sigma}^{2}-m_{\sigma}^{2}\right)^{2}\right]
$$

$M=\tilde{\sigma} U$ is the Higgs Field. $\sigma=\tilde{\sigma}-m_{\sigma}$ is the physical Higgs. The semi-classical limit is $g^{2}$ small, $m_{\sigma}^{2}$ and $\kappa=\frac{m \omega}{m \sigma}$ fixed.

The Euclidean equations of motion are:

$$
\begin{aligned}
& D_{\mu} W_{\mu \nu}+\frac{i \kappa^{2}}{2}\left(D_{\nu} M\right)^{\dagger} M-\frac{i \kappa^{2}}{2} M^{\dagger} D_{\nu} M=0 \\
& D_{\mu}^{\dagger} D_{\mu} M+\left(\frac{1}{2} \operatorname{Tr}\left(M^{\dagger} M\right)-m_{\sigma}^{2}\right) M=0
\end{aligned}
$$

with no source terms $(\lambda=0$ in (1.1)) there's a finite action solution:

$$
\begin{aligned}
W_{\mu}^{0} & =\frac{2 \rho^{2}}{x^{2}\left(x^{2}+\rho^{2}\right)} x_{\nu} \bar{\tau}_{\mu \nu} \\
M^{0} & =\left(\frac{x^{2}}{x^{2}+\rho^{2}}\right)^{1 / 2} m_{\sigma} \mathbb{1}
\end{aligned}
$$

However, the full action (2.4) is infinite for this configuration. Therefore we try to deform (2.6) to get another finite action solution. Try:

$$
\begin{aligned}
M & =M^{0}+\delta M \\
W_{\mu} & =W_{\mu}^{0}+\delta W_{\mu}
\end{aligned}
$$

We get equations of the form

$$
\mathcal{O} \delta \phi=J
$$

Where $\phi$ is either $W_{\mu}$ or $M, \mathcal{O}$ is some differential operator. $\delta \phi$ is also subjected to some boundary conditions to ensure a finite action (namely $\delta \phi \rightarrow 0$ fast enough at infinity).

The two above conditions on $\delta \phi$ are inconsistent. The operator $\mathcal{O}$ has zero modes, so the source $J$ propagates to infinity and determine the boundary values of $\delta \phi$. These boundary values correspond to the required ones only if $J$ is perpendicular to the zero modes of $\mathcal{O}$. 
Resolution of this problem was given in 44. Constraining the path integral in a particular way has the effect of adding operators to the Lagrangian. The modified Lagrangian is called the constrained Lagrangian. The new operators are required to vanish fast enough at infinity (faster than $\frac{1}{|x|^{4}}$ for the configuration (2.6)) so they don't change the finite action boundary conditions. However, they do change the source $J$ in (2.8), and can be tuned to make it perpendicular to the zero modes. Therefore, one can get instanton solutions to the constrained Lagrangian, which are approximate solutions to the original Lagrangian.

Asymptotic expressions for the resulting configuration can be obtained in two different regions [4, 6]. First, in the unbroken phase $\left(|x|<v^{-1}\right)$ we expect to get approximately an 'tHooft instanton of size $\rho$, which solves the equations:

$$
\begin{array}{r}
D_{\mu} W_{\mu \nu}=0 \\
D^{2} M=0
\end{array}
$$

These equations cease to be a good approximation to the full equations of motion where the neglected terms start to dominate. This determines the range of validity of $(2.9)$. One gets:

$$
\begin{aligned}
W_{\mu} & =\frac{2 \rho^{2}}{x^{2}\left(x^{2}+\rho^{2}\right)} x_{\nu} \bar{\tau}_{\mu \nu}+\cdots & & |x| \ll m_{\omega}^{-1} \\
M & =\left(\frac{x^{2}}{x^{2}+\rho^{2}}\right)^{1 / 2} m_{\sigma} \mathbb{1}+\cdots & & |x| \ll m_{\sigma}^{-1}
\end{aligned}
$$

To get an asymptotic expression for $|x| \gg \rho$ one leaves in the equations of motion just the leading terms at infinity (For the configurations (2.10)) [ [4] All other terms in (2.5) are replaced by a delta function source. The resulting equations are:

$$
\begin{aligned}
& \partial^{2} M-m_{\sigma}^{2} M \propto \delta^{(4)}(x) \\
& \partial_{\mu} W_{\mu \nu}+i \kappa^{2} m_{\sigma}^{2} W_{\nu} \propto \delta^{(4)}(x) .
\end{aligned}
$$


The terms in (2.11) vanish as $\frac{1}{|x|^{4}}$ as $|x| \rightarrow \infty$, whereas all the other operators are suppressed by powers of $\frac{\rho^{2}}{|x|^{2}}$, including the terms in the constrained Lagrangian added to impose the constraints.

This gives the asymptotic expansion:

$$
\begin{aligned}
W_{\mu} & =-\rho^{2} \bar{\tau}_{\mu \nu} \partial_{\nu} G\left(m_{\omega},|x|\right)+\cdots \\
M & =m_{\sigma} \mathbb{1}\left(1-\frac{1}{2} \rho^{2} G\left(m_{\sigma},|x|\right)+\cdots\right) \quad|x| \gg \rho
\end{aligned}
$$

where

$$
\begin{aligned}
& G(m,|x|)=m^{2} \frac{K_{1}(m|x|)}{m|x|} \\
& \left(\square-m^{2}\right) G(m,|x|)=-4 \pi^{2} \delta(x) .
\end{aligned}
$$

One assumes $\rho v \ll 1$, large instantons cannot be treated that way to yield an approximate saddle point. The above expressions are first order in the small parameter $\rho v$. Since $\rho \ll v^{-1}$ the regions of the above expressions are overlapping, so the configuration is well defined everywhere. The two expressions have to be patched in the intermediate region $\left(\rho<|x|<v^{-1}\right)$.

The fermionic zero modes of this configuration have similar expansions [6]. It is also assumed that $\mathcal{M}_{q}<v$, which is the case considered in this paper.

\section{The low energy effective Lagrangian:}

Consider the energy range $m_{\omega}<E<m_{t}<v$. In this range all virtual configurations smaller than $m_{t}^{-1}$ should be expressed as a series of local operators. This includes perturbative configurations (like heavy quark loops) as well as small instantons. Therefore the 't Hooft operator should be included in the effective Lagrangian, as a part of the matching process.

The 't Hooft operator involves necessarily a heavy quark. Therefore its not part of the physics to be described by the low energy effective theory. However, with family mixing there are low energy baryon number violating operators, 
resulting from virtual heavy quarks. An example of a dimension 21 operator is given in Figure 1. This operator and similar ones should be a part of the effective Lagrangian.

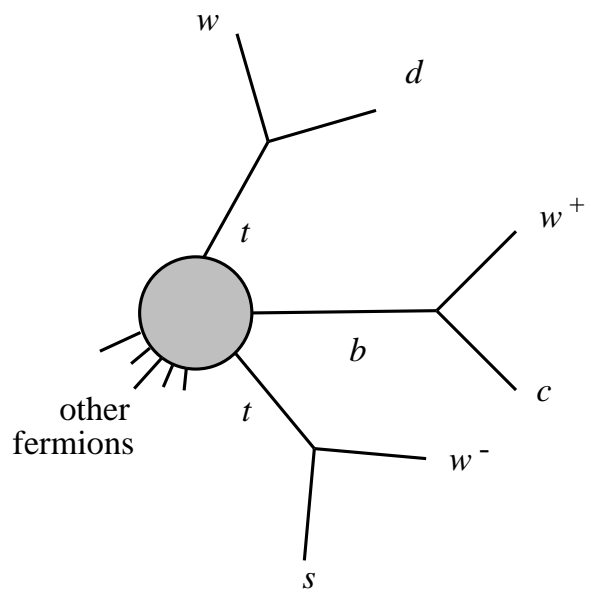

(a) In the full theory

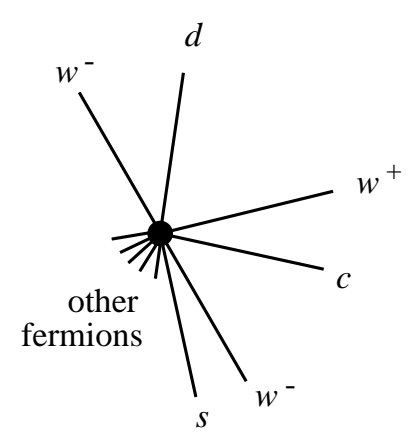

(b) In the effective theory

Figure 1

The situation is similar in the case of integrating out just the top (assuming $\left.m_{t} \gg m_{b}\right)$. The resulting theory is non-linearly realized [7, 8]. Some of the fermions transform linearly under $\mathrm{SU}(2)$, and the bottom transforms nonlinearly, as a part of an incomplete multiplet. To build $\mathrm{SU}(2)$ invariants it's convenient to use the composite field $U\left(\begin{array}{l}0 \\ b\end{array}\right)$ which transform linearly as an $\mathrm{SU}(2)$ doublet [9].

Expanding $U=\exp \left(i \frac{\zeta^{a} \tau^{a}}{v}\right)$ shows that replacing the complete doublet by $U\left(\begin{array}{l}0 \\ b\end{array}\right)$ in the 't Hooft operator recovers the original operator with $b$ emitted, but also a series of operators involving $\zeta^{a}$ (the longtitudal gauge bosons). These additional operators are suppressed by powers of $v^{-1}$, and represent effects of integrating out the top. 


\section{Instanton Suppression in the effective theory:}

An instanton solution to the low energy theory, and corresponding fermionic zero modes for the remaining two quark doublets would contradict the full theory's predictions and therefore violate decoupling. We show now that the low energy theory doesn't admit instantons.

Integrating out heavy fermions induces a series of higher dimensional terms in the effective Lagrangian, see for example [10, 9]. It was suggested [2] that some of these operators give rise to infinite action (zero measure) for the original constrained instanton. However, the divergences come from the short distance behavior of these operators, where the effective action cannot be used.It is also conceivable that one can modify the original solution (by changing the constraints, for instance) to another approximate solution which accommodates the higher dimensional terms and has a finite action. This would represent threshold corrections to the instanton coming from integrating out the fermions.

In the following we demonstrate that no such solution is possible. We assume, ex absurdo, that the constrained Lagrangian can be adjusted to enable finite (effective) action boundary conditions. We study the resulting asymptotic expression for $|x| \gg \rho$ and conclude that it cannot be patched to any configuration with a non-zero winding number, thus contradicting the assumption.

Assume then that we have an instanton configuration (2.10) at short distances. We now try to get an asymptotic expression similar to (2.12). Most new operators in the effective theory will be replaced by a delta function source in (2.11), but some will also contribute to the left hand side of equation (2.11). Consider for example the operator:

$$
\Delta \mathcal{L}=\frac{1}{4 \pi^{2} m_{\sigma}} \sigma \operatorname{Tr}\left(W_{\mu \nu} W_{\mu \nu}\right)
$$


coming from a diagram shown in figure (2).

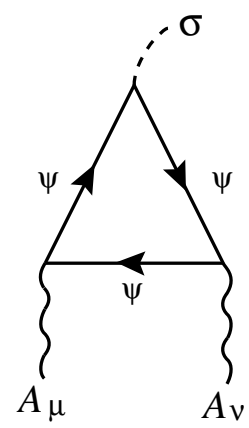

Figure 2

This operator cannot be ommited in the region described by equation (2.11), since it dominates the existing terms for $|x|<\frac{\rho}{\sqrt{\rho v}}$. In particular we get the modified equation :

$$
\kappa^{2}\left(\square-m_{\sigma}^{2}\right) \sigma+\frac{1}{4 \pi^{2} m_{\sigma}} \operatorname{Tr}\left(W_{\mu \nu} W_{\mu \nu}\right)=2 \pi^{2} \rho^{2} \delta(x) .
$$

All other higher dimensional operators in the equations of motion are suppressed by powers of $\frac{\rho^{2}}{|x|^{2}}$ or $\left(m_{t}|x|\right)^{-1}$. Therefore equation (4.2) holds for $|x| \gg m_{t}^{-1}$ (not for all $|x| \gg \rho$ as in section 2).

Equation (4.2) is an equation of the form (2.8), which in general imposes restrictions (orthogonality conditions) on the source. To see these restrictions multiply (4.2) by $G\left(m_{\sigma},|x|\right)$ and integrate from $r>m_{t}^{-1}$ to infinity. The left hand side picks up just boundary terms at $r$. Choosing $r<m_{\sigma}^{-1}$ and $r<m_{\omega}^{-1}$, the unbroken solution (2.10) still holds. Therefore:

$$
\frac{1}{v} \int_{r}^{\infty} d^{4} x \operatorname{Tr}\left(W_{\mu \nu} W_{\mu \nu}\right) G\left(m_{\sigma},|x|\right) \sim 0\left(v \frac{\rho^{2}}{r^{2}}\right) .
$$

(Using: $\left.G\left(m_{\sigma},|x|\right) \sim \frac{1}{|x|^{2}}+0(\rho v)\right)$

or:

$$
W_{\mu \nu}(|x|=r) \ll 0\left(\frac{\rho v}{r^{2}}\right) .
$$

This statement is modified by corrections to (2.10), (4.3) etc. However, all these corrections are of order $\rho v$. To first order in $\rho v$, (4.4) implies that 
$W_{\mu \nu}=0$ for $m_{t}^{-1}<r<m_{\omega}^{-1}$. This is inconsistent with the assumption that we have an instanton in the unbroken phase.

The corrections mentioned above make $W_{\mu \nu} \sim 0(\rho v)$. This would give winding number of order $\rho v$. However, since $\rho v \ll 1$, this is still inconsistent with having an instanton configuration, small corrections cannot generate a winging number. Therefore we conclude that there are no possible approximate instanton solutions in the effective theory.

\section{Conclusions}

In this paper we have considered small instantons coupled to fermions lighter than the symmetry breaking scale. We have integrated out one heavy quark doublet. In this limit we have shown that there are no instantons in the low energy theory. The result came from considering the region where an instanton is patched to an asymptotic expression. Integrating out the fermions changes the asymptotic expression, making it impossible to patch it to an instanton. Even though the core of the instanton is not describable by the effective theory, our considerations relied only on regions where the effective theory is valid. This was possible due to the existence of an operator in the effective theory that is not suppressed by powers of the heavy fermion mass, and therefore can affect the region describable by the effective theory.

\section{Acknowledgements}

The author wishes to thank W. Fischler for suggesting the problem, for his invaluable advice and criticism and for his comments on the manuscript. Useful conversations with J. Distler, J. Feinberg, S. Paban and J.M. Rodriguez are gratefully acknowledged. The author also thanks T. Gould for a useful correspondence. 


\section{Appendix}

The standard model action can be obtained from the general theory of nonlinear realization [7], see for example [9].

The bosonic Lagrangian is

$$
\mathcal{L}_{B}=-\frac{1}{2} \operatorname{Tr}\left(\hat{W}_{\mu \nu} W^{\mu \nu}+\hat{B}_{\mu \nu} \hat{B}^{\mu \nu}\right)+\frac{1}{2}\left(D_{\mu} M^{\dagger} D^{\mu} M\right)+V\left(M^{\dagger} M\right)
$$

$W_{\mu}, B_{\mu}$ are $\mathrm{SU}(2)$ matrices

$$
\begin{aligned}
\hat{W}_{\mu} & =W_{\mu}^{a} t^{a} \\
\hat{B}_{\mu} & =B_{\mu} t^{3}
\end{aligned}
$$

$M=\tilde{\sigma} U$, the covariant derivatives are:

$$
\begin{aligned}
D_{\mu} \tilde{\sigma} & =\partial_{\mu} \tilde{\sigma} \\
D_{\mu} \hat{U} & =\partial_{\mu} \hat{U}+i g \hat{W}_{\mu} \hat{U}-i g^{\prime} \hat{U} \hat{B}_{\mu} .
\end{aligned}
$$

The top-bottom Lagrangian is:

$$
\mathcal{L}_{\psi}=i \psi^{\dagger} \sigma^{\mu} D_{\mu} \psi+i \bar{\psi}^{\dagger} \sigma^{\mu} D_{\mu} \bar{\psi}-\left[\bar{\psi}^{T} \frac{M_{q}}{v} M \psi+\text { c.c. }\right]
$$

All the Fermions used are left handed Weyl Fermions. The covariant derivatives are:

$$
\begin{aligned}
& D_{\mu} \psi=\left(\partial_{\mu}+i g W_{\mu}+\frac{2 i}{3} g^{\prime} t_{3} \hat{B}_{\mu}\right) \psi \\
& D_{\mu} \psi=\left(\partial_{\mu}-i g^{\prime}\left(1+\frac{2 t_{3}}{3}\right) B_{\mu}\right) \bar{\psi}
\end{aligned}
$$




\section{References}

[1] T. Banks and A. Dabholhar, Phys. Rev. D46 (1992) 4016 .

[2] H. Georgi, L. Kaplan and D. Morin, Phys. Rev. D49 (1994) 2457 .

[3] S. Hsu, Harvard University Preprint, HUTP-93/A021.

[4] I. Affleck, Nucl. Phys. B191 (1981) 429 ;

I. Affleck, M. Dine and N. Seiberg, Nucl. Phys. B241 (1984) 493 .

[5] G. 't Hooft Phys. Rev. Lett. 37(1976) 8, Phys. Rev. D14 (1976) 3431 , Phys. Rev. D18 (1978) 2199 .

[6] O. Espinosa, Nucl. Phys. B343 (1990) 310 ;

A. Ringwald, Nucl. Phys. B330 (1990) 1 .

[7] S. Coleman, J. Wess and B. Zumino, Phys. Rev. 177 (1969) 2239.

[8] F. Feruglio, A. Masiero and L. Maiani, Nucl. Phys. B387 (1992) 52 .

[9] F. Feruglio, Int. J. Mod. Phys. A8 (1993) 4937 .

[10] E. D’Hoker and E. Farhi, Nucl. Phys. B248 (1984) 59,77 . 